\title{
Discriminating patients with paediatric idiopathic hyperhidrosis from healthy subjects with infrared thermography and machine learning classifiers
}

\author{
by F. Carvalho*,**, R. Vardasca***,****, C. Magalhaes ${ }^{* * *},{ }^{* * * *}$, J. Mendes ${ }^{* * *,{ }^{* * *}}$ and J. Goncalves ${ }^{* * * * *}$ \\ * Centro Materno Infantil do Porto, Centro Hospitalar e Universitário do Porto, Largo da Maternidade de Júlio \\ Dinis, 4050-651 Porto, Portugal, fmfscc@gmail.com \\ ** Instituto de Ciências Biomédicas Abel Salazar, Universidade do Porto, Rua Jorge de Viterbo Ferreira 228, \\ 4050-313 Porto, Portugal \\ *** Faculdade de Engenharia, Universidade do Porto, Rua Dr Roberto Frias, 4200-465 Porto, Portugal, \\ ricardo.vardasca@fe.up.pt \\ **** INEGI, Universidade do Porto, Rua Dr Roberto Frias 400, 4200-465 Porto, Portugal \\ **** Faculdade de Farmácia, Universidade do Porto, Rua Jorge de Viterbo Ferreira 228, 4050-313 Porto, \\ Portugal
}

\section{Abstract}

Idiopathic hyperhidrosis $(\mathrm{IH})$ is a medical condition characterised by excessive sweating beyond what is physiologically necessary for thermoregulation affecting mainly the axillae and palms. It affects seriously the quality of life of patients and has an incidence at paediatric age of $1.6 \%$. The diagnosis is subjective relying only the the patient claim and physician perception. It is aim of this research to evaluate if dynamic infrared thermography (IRT) along with machine learning classifier on the thermal data are able to discriminate $\mathrm{IH}$ paediatric patients from healthy subjects. Using dynamic IRT, through convective provocation, on the views of axillae and palms, it was possible to discriminate IH paediatric patients from healthy subjects using artificial neural networks (ANN) and random forests (RF) in thermal measurements with high accuracy $(>99 \%)$, the same was not possible only with the thermal data and statistics.

\section{Introduction}

Idiopathic hyperhidrosis $(\mathrm{IH})$ is defined as perspiration in excess of the physiologic amount necessary to maintain thermal homeostasis. The prevalence of IP is underestimated by the currently reported figures, this due to underreported claims by patients and under-diagnosed by physicians, especially in the paediatric population, which is estimated to be around $1.6 \%$ [1]. This condition affects seriously the patients quality of life and self-esteem.

The lack of objective assessment methods to aid in the diagnosis acts as a major barrier for health professionals, this is also true on the assessment of available treatments. Attention is required to address this.

Infrared thermography (IRT) has been used to document a single case of unilateral localised IH over left scalp and face [2], identifying the affected areas. It was also investigated as outcome measure to the IH treatment by thoracic sympathetic surgery $[3,4]$, in which it corroborated with the patients feedback, demonstrating the potential of this imaging technique. Although standardisation in views and data collection procedure, which was mainly static, are required for deeper interpretation of the physiological states. Dynamic IRT has showed to provide more physiological information about autonomous nervous system than static IRT [5]. The usage of machine learning classifiers also demonstrated to improve the usage of the data provided by this imaging technique $[6,7]$.

It is aim of this research to evaluate if infrared dynamic IRT along with machine learning classifier on the thermal data are able to discriminate $\mathrm{IH}$ paediatric patients from healthy subjects

\section{Methodology}

The methodology used in the research is divided in the participants recruiting and screening and the data analysis and classification.

\subsection{Data collection}

The participants used in this research were patients of the paediatric surgery department of Centro Materno Infantil do Porto at Centro Hospitalar e Universitário do Porto. In total were screened 41 paediatric IH patients clinically diagnosed and 9 healthy paediatric subjects, all signed the informed consent before taking part in the study, which was approved by the hospital ethical committee.

The participants underwent to a temperature controlled room, disrobed the clothing to underwear and acclimatised for 10 minutes at room initial temperature $20^{\circ} \mathrm{C}$ a set of images from axillae and palms were taken using a FLIR E60 thermal camera (FPA sensor size of $320 \times 240$, NETD $<50 \mathrm{mK}$ and measurement uncertainty of $\pm 2 \%$ of the overall temperature reading), then the room was heated using 4 convectors of $200 \mathrm{~W}$ each, lined in two at each side, $1 \mathrm{~m}$ of the patient seated in a bench to the room temperature of $24^{\circ} \mathrm{C}$ and the set of thermal images was repeated. 


\subsection{Data analysis}

There were defined regions of interest (ROI) for the both palms and axillae, from which the mean temperature was recorded, being the images taken at $20^{\circ} \mathrm{C}$ considered baseline and at $24^{\circ} \mathrm{C}$ considered as after thermal provocation, temperature differences were calculated per ROI considering after provocation minus baseline $(\Delta T)$. These results were statistically evaluated using the non-parametric test Mann-Whitney $U$ considering the $95 \%$ interval of confidence. The dataset was loaded in the WEKA machine learning simulation tool [8] and it was split into two datasets: training (70\% of the sample) and testing (30\% of the sample), the machine learning classifiers tested were: artificial neural networks (ANN), k-Nearest Neighbour with 3 (kNN3) and 5 (kNN5) neighbours, Random Forests (RF) and Support Vector Machines (SVM). These were evaluated using the precision, recall, F-Score and Accuracy.

\section{Results}

The variation in mean temperature at the ROls is shown at the Fig. 1 , it can be observed that the obtained values with their variation overlaps the two groups of the sample, this is in line with what the statistics showed, no evidence of discrimination between the groups (Mann-Whitney $U, p>0.05$ ). Although using the different ROls as entrances to the ML classifiers, $100 \%$ accuracy was obtained with ANN and RF for both axillae and palms, also with kNN3 for axilla, the worst performance was obtained by SVM.

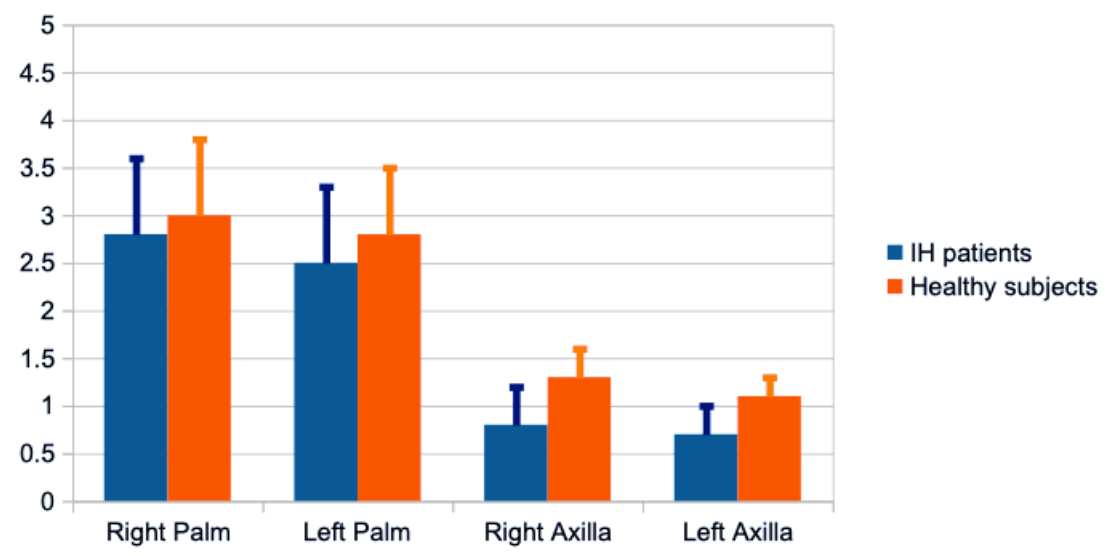

Fig. 1. The $\Delta T$ of the mean temperature of the ROls from baseline.

Table 1. The results of classification of the two groups of the sample using ML classifiers in thermal data.

\begin{tabular}{|l|c|c|c|c|c|c|c|c|}
\hline \multirow{2}{*}{ Classifiers } & \multicolumn{4}{|c|}{ Axillae } & \multicolumn{4}{c|}{ Palms } \\
\cline { 2 - 9 } & Accuracy & Precision & Recall & F-Score & Accuracy & Precision & Recall & F-Score \\
\hline ANN & $100.0 \%$ & 1.000 & 1.000 & 1.000 & $100.0 \%$ & 1.000 & 1.000 & 1.000 \\
\hline kNN3 & $100.0 \%$ & 1.000 & 1.000 & 1.000 & $93.3 \%$ & 0.923 & 0.938 & 0.933 \\
\hline kNN5 & $86.6 \%$ & 0.867 & 0.867 & 0.867 & $93.3 \%$ & 0.938 & 0.938 & 0.933 \\
\hline RF & $100.0 \%$ & 1.000 & 1.000 & 1.000 & $100.0 \%$ & 1.000 & 1.000 & 1.000 \\
\hline SVM & $86.6 \%$ & 0.928 & 0.867 & 0.867 & $86.6 \%$ & 0.928 & 0.867 & 1.000 \\
\hline
\end{tabular}

\section{Discussion and conclusion}

From only thermal values and with statistics there is no possibility of discriminating both groups at the sample in static and dynamic IRT. However using this values as entrances to ML classification algorithms, those showed their importance in aiding the discrimination adding decision value to the thermal data, this is in line with the biomedical 
applications described by Vardasca et al. in 2019 [7]. Also the usage IRT in IH at clinical setting is promising as initially suggested by pioneer studies [2-4].

It was possible to discriminate $\mathrm{IH}$ paediatric patients from healthy subjects using artificial neural networks (ANN) and random forests (RF) in thermal measurements with high accuracy $(>99 \%)$, this tools can be used together aiding daly diagnosis of IH patients,

\section{ACKNOWLEDGEMENTS}

The authors thank the partial funding of the Project LAETA - UID/EMS/50022/2013.

\section{REFERENCES}

1. Gordon, J. R., Hill, S. E. "Update on pediatric hyperhidrosis". Dermatologic therapy, 26(6), 452-461, 2013.

2. Lim, H. S., Whang, S. W., Yoon, D. H., Lee, M. G. "Unilateral Localized Hyperhidrosis Over Scalp and Face". Korean Journal of Dermatology, 39(9), pp. 1000-1002, 2001.

3. Choi, S. H., Lim, Y. H., Lee, S. Y., Choi, J. B. "Relation between changes of DITI and clinical results according to the level and extent of sympathicotomy in essential hyperhidrosis". Korean J Thorac Cardiovasc Surg, 37(1), pp. 64, 2004.

4. Haam, S. J., Park, S. Y., Paik, H. C., Lee, D. Y. "Sympathetic nerve reconstruction for compensatory hyperhidrosis after sympathetic surgery for primary hyperhidrosis". Journal of Korean medical science, 25(4), pp. 597-601, 2010.

5. John, H. E., Niumsawatt, V., Rozen, W. M., Whitaker, I. S. "Clinical applications of dynamic infrared thermography in plastic surgery: a systematic review". Gland surgery, 5(2), pp. 122, 2016.

6. Vardasca, R., Vaz, L., Mendes, J. "Classification and Decision Making of Medical Infrared Thermal Images". In Classification in BioApps. Springer, Cham, pp. 79-104, 2018.

7. Vardasca, R., Magalhaes, C., Mendes, J. "Biomedical Applications of Infrared Thermal Imaging: Current State of Machine Learning Classification”. In Multidisciplinary Digital Publishing Institute Proceedings, Vol. 27, No. 1, pp. 46, 2019.

8. Hall, M., Frank, E., Holmes, G., Pfahringer, B., Reutemann, P., Witten, I. H. "The WEKA data mining software: an update". ACM SIGKDD explorations newsletter, 11(1), pp. 10-18, 2009. 